\title{
MedienPädagogik
}

Zeitschrift für Theorie und Praxis der Medienbildung

Jahrbuch Medienpädagogik 3.

Zweitveröffentlichung aus: Jahrbuch Medienpädagogik 3. (2003) Opladen: Leske + Budrich. Herausgegeben von Ben Bachmair, Peter Diepold und Claudia de Witt.

\section{Online-Ethnographie - Wege und Ergebnisse zur Forschung im Kulturraum Internet}

\author{
Winfried Marotzki
}

Im vorliegenden Beitrag wird der Begriff der Online-Ethnographie eingeführt, indem zunächst die zugrunde liegende doppelte Transformation der klassischen Ethnographie dargestellt wird. Im zweiten Schritt wird der Gegenstandsbereich der Online-Ethnographie auf virtuelle Gruppen spezifiziert und schließlich - drittens - acht Merkmalsbereiche virtueller Gruppen herausgearbeitet.

\section{Die doppelte Transformation der klassischen Ethnographie}

Mit der Entwicklung des Internet eröffnet sich ein neuer Kulturraum, innerhalb dessen der Mensch differenziertere Möglichkeiten hat, ein Verhältnis zu sich und zur Welt aufzubauen (vgl. Sandbothe/Marotzki 2000). In diesen neuen Möglichkeiten der Selbst- und Weltreferenz liegt die bildungstheoretische Bedeutung des Kulturraums Internet (vgl. Marotzki 2001). Es ist unter anderem diese bildungstheoretische Relevanz, die das neue Medium Internet zu einem wichtigen Gegenstand qualitativer Forschung macht und die Frage aufwirft, wie der Kulturraum Internet zu erforschen ist.

Zunächst gilt, was auch für andere uns neue und fremde Kulturräume gilt: Nähert man sich nämlich einem anderen Kulturraum in ethnographischer Absicht, so werden zunächst soziale Strukturen, Regeln, Konventionen, Interaktionen, Kommunikationsstrukturen und Gruppenbildungen interessieren. Man wird Dokumente dieses Kulturraums untersuchen und selbst in ihn hineingehen, um zu beobachten und mitzuerleben, was dort in welcher Weise geschieht und wie Kultur strukturiert ist. Zinnecker beschreibt diesen Ansatz der klassischen Ethnographie wie folgt: „Ein humanwissenschaftlich ausgebildeter Experte besucht auf Zeit eine lokale Lebenswelt, die zum Besuchszeitpunkt nicht seine eigene ist. Er tut das in der Absicht, darüber einen Bericht zu veröffentlichen. Der Bericht soll die Rahmenbedingungen dieses kulturellen Handlungsfeldes benennen, die typischen Handlungspraxen der 
Lebenswelt-Bewohner dokumentieren und schließlich den Sinnhorizont der dort zusammenlebenden Menschen - aus deren Perspektive - erschließen. Die vorwiegende Forschungstätigkeit des Ethnographen besteht in der teilnehmenden Beobachtung der Praxen und Gespräche im Feld. Diese wird ergänzt durch Interviews, beiläufige wie formelle, mit Bewohnern, insbesondere mit Schlüssel-Informanten. Beobachtungen und Gespräche werden während des Feldaufenthaltes in Forschungs-Tagebüchern protokolliert und zwischenausgewertet. Die beiden Tätigkeiten werden ergänzt durch Recherchen zu kulturellen Dokumenten, die im Rahmen der lokalen Lebenswelt - und gegebenenfalls der gleichen Lebenswelt andernorts - produziert wurden und werden“ (Zinnecker 2000, S. 382).

Dieser klassische Ansatz der Ethnographie, der anhand der Exploration geographisch anderer Kulturen u.a. von Bronislaw Malinowski (vgl. Malinowski 1922) entwickelt worden war, ist dann - das wäre die erste Transformation - zum Zwecke der Exploration von (Teil)Kulturen im eigenen Lande weiter entwickelt worden (vgl. dazu Friebertshäuser 2000). Der Blick, wie er auf andere Kulturen gerichtet wurde, ist mit dem gleichen Setting auf die eigene Kultur gelenkt worden. Zinnecker spricht treffend von der „Rückwendung des ethnographischen Blicks auf das Fremde in der eigenen Kultur“ (Zinnecker 2000, S. 383).

Die zweite Transformation besteht nun darin, die neuen Kulturräume des Internet zu explorieren, d.h. den ethnographischen Blick auf virtuelle Welten zu richten ${ }^{1}$. Eine solche ethnographische Herangehensweise an den neuen Kulturraum Internet ist als OnlineEthnographie bezeichnet worden (Paccagnella 1997; Hine 1998). Online-Ethnographie hat den Fokus auf Gruppenbildungen, die sich im Internet vollziehen und insofern Sozialität und Kulturalität konstituieren (Forschungsfokus: Online). Sie kann systematisch von zwei verwandten, aber doch differenten Forschungsfoki unterschieden werden.

1 Ein weiteres Beispiel für eine frühe kulturtheoretische Sichtweise des Internet: das Projekt „Interaktionsraum Internet. Netzkultur und Netzwerkorganisation in offenen Datennetzen“ ist an der TU Berlin in den Jahren 1996-98 von der Projektgruppe „Kulturraum Internet“ durchgeführt worden. Gefördert wurde es von der Volkswagen-Stiftung im Rahmen des Schwerpunkts „Neue Informations- und Kommunikationstechniken in Wirtschaft, Medien und Gesellschaft: Wechselwirkungen und Perspektiven“. Abschlußbericht 1998 (http:// duplox.wz-berlin.de/endbericht/, 26.09.2000). Elizabeth Reid hat ebenfalls sehr früh, bereits 1994, auf die Kulturdimension des Internet aufmerksam gemacht (vgl. Reid 1994). 


\section{Internetforschung: Grundlegende Forschungsfoki}

\subsection{Forschungsfokus: Offline}

Zum einen können die Nutzer des Internet und ihre Lebenswelten offline untersucht werden: Wie sie mit dem Medium umgehen und wie sie es in ihre Lebenswelten integrieren, kann Gegenstand der Forschung sein (siehe dazu Vogelgesang u.a. 1991; Vogelgesang 1994). Der Mediengebrauch im Kontext der alltäglichen Lebenswelt steht hier also im Zentrum der Aufmerksamkeit. Methodisch werden hier Befragungen, z.B. Interviews, zum Einsatz kommen, die durchaus auch Online durchgeführt werden können (vgl. Bandilla 1999). Solche Befragungen können sich beispielsweise darauf richten, wie oft die Befragten im Netz sind, ob sie insgesamt in dem Maße weniger Fernsehen schauen, wie sie ins Internet gehen, oder allgemein: welche Bedeutung ihre Internetaktivitäten für ihre Freizeit, fürs Lernen, für ihren Beruf oder ggf. für ihre Familie haben.

Miller und Slater (2000) untersuchen beispielsweise den Internetgebrauch auf der karibischen Insel Trinidad und zeigen, wie sich dadurch gesellschaftliche, soziale, familiale und biographische Strukturen (zum Positiven) verändern. Die Autoren beschreiben dezidiert, wie das Internet integraler Bestandteil der genannten Ebenen wird. Sie weisen explizit Standpunkte zurück, die - wie beispielsweise Manuell Castells mit seiner Unterscheidung von the self und the net (vgl. Castells 1996) - von einer Trennung der realen Welt von einer virtuellen ausgehen. Die Aktivität „doing Internet“ impliziere, dass diese Aktivität immer lebensweltlich verankert sei und als kommunikative, informative oder selbstpräsentative Aktivität wie jede andere auch zu sehen sei. Die Autoren gehen also systematisch nicht davon aus, dass sich auf der einen Seite Trinidad mit seiner spezifischen Kultur befinde und nun zu schauen ist, was das Internet mit dieser Kultur macht, sondern sie schauen sich die sozialen Akteure und deren Umgehen mit dem Internet an. Insofern widmen sich die Autoren dem Eingebundensein des Internet in einer bestimmten Kultur. Es handelt sich gerade deshalb um eine sehr interessante Studie, da sie zu dem Resultat kommt, dass das neue Medium den Einwohnern Trinidads die Möglichkeit gibt, sich als Trinidaner zu identifizieren, d.h. Ethnizität wird durch das Internet in neuer Weise konstituiert (vgl. auch Zurawski 2000).

\subsection{Forschungsfokus: Online-Offline}

Zweitens kann das Verhältnis von Online und Offline untersucht werden, d.h. es wird untersucht, was von Einzelnen und/oder Gruppen Online an Aktivitäten entfaltet wird und wie diese im Verhältnis zu ihrer Lebenswelt stehen. James Slevin (2000) beschreibt in seinem Buch, wie das Internet gesellschaftliche, institutionelle, politische, ökonomische, soziale und kulturelle 
Bereiche transformiert. Das Internet-Journal IT\&Society (http://www.stanford.edu/ group/siqss/itandsociety/ [28.09.02]) widmet sich programmatisch der Kraft, die das Internet auf Lebenswelten und die ganze Gesellschaft ausübt: „IT \& Society is a webbased scholarly joumal devoted to the scientific analysis of the social impact of information technology on society, with special emphasis on quantitative survey analysis“. Hier finden sich Analysen der Auswirkung des Netzes auf praktisch alle Lebensbereiche: vom Zeitverständnis über das Familienleben bis hin zur Sexualität. In gewisser Weise kann man sagen, dass es sich hier um Wirkungsforschung im klassischen Sinne handelt. An dieser Stelle soll nicht diskutiert werden, wie sich diese Art der Wirkungsforschung zu den kritischen Debatten um Wirkungsforschung im Kontext der Medienpädagogik verhält (vgl. Kübler 1995).

\subsection{Forschungsfokus: Online}

$\mathrm{Zu}$ jenen Forschungen, die dezidiert einen Online-Forschungsfokus aufweisen, gehören zunächst alle Forschungen, die sich auf das internetinterne Nutzerverhalten in der Absicht beziehen, aus diesen Analysen etwa im Rahmen von E-Commerce bessere Vermarktungschancen und -Strategien für die eigenen Produkte innerhalb des Internet zu entwickeln. Zur Analyse des Nutzerverhaltens im Kontext einer solchen Marktforschung sind einige spezielle Methoden entwickelt worden: (1) Über logfile-Analysen kann ermittelt werden, welche Inhalte wie oft abgerufen werden. (2) Das User Tracking zeichnet die Wege des Nutzers auf und gibt so Aufschluss über die Vorlieben, Verweildauer und anderes Verhalten. Es erlaubt beispielsweise zielgenaue Werbung. Eine Variante davon ist das Echtzelt Tracking, das ebenfalls die Wege aufzeichnet; abhängig davon wird unmittelbar entsprechende Werbung generiert: activity-response-Werbung. (3) Unter Collaboratlve Filtering versteht man, dass beispielsweise mittels der Software Net Perceptlon von Eigenschaften einer Käufergruppe auf andere Eigenschaften dieser Käufergruppe geschlossen wird, indem man sie mit einer anderen Käufergruppe mit ähnlichen Merkmalen vergleicht. Wenn Person A beispielsweise im Netz Jazz-CDs bestellt, dann ermittelt die Software, dass die meisten, die Jazz-CDs bestellt haben, auch Rotweintrinker sind. Also lohnt es sich, diesem Kunden Rotwein anzubieten. Ein gutes Beispiel für Collaborative Filtering findet sich bei dem Online Buchhändler Amazon: Wenn man ein Buch geordert hat, erscheint die Rubrik „Kunden, die dieses Buch gekauft haben, haben auch folgende Bücher gekauft“. Die Diskussion in der deutschen Mailingliste gir-l (German Internet Research) vermittelt einen guten Überblick über die Bandbreite der hier untersuchten und bearbeiteten Phänomene (vgl. auch Reips u.a. [Hrsg.] 2000; weiterhin: www.dgof.de [Deutsche Gesellschaft für Online-Forschung]).

Im Gegensatz zu einer an der Marktforschung angelehnten Internet-Forschung, die nicht nur, aber doch überwiegend ein quantitatives Forschungs- 
design favorisiert (vgl. Batinic u.a. [Hrsg.] 1999) und schwerpunktmäßig am Nutzerverhalten interessiert ist, zeichnet sich der Zugang einer Online-Ethnographie durch zwei Merkmale aus: Er arbeitet mit qualitativen Forschungsansätzen und bezieht sich überwiegend auf virtuelle Communities im Internet. Einen ersten guten Methodenüberblick haben Chris Mann und Fiona Stewart (vgl. Mann/Stewart 2000) gegeben. Sicherlich werden in den nächsten Jahren auf diesem Gebiet noch weitere Innovationen der Methodenentwicklung stattfinden. In der vorliegenden Arbeit möchte ich die Methodenfrage nicht in das Zentrum stellen, sondern versuchen, den Gegenstandsbereich, nämlich virtuelle Communities, näher zu bestimmen.

\section{Virtuelle Communities}

Die Forschung zu virtuellen Communities entwickelte sich parallel zur Entstehung des Internet. Während das ARPANET (Vorgänger des Internet) ursprünglich dafür entwickelt worden war, Computer miteinander zu verbinden, verdankte es seinen durchschlagenden Erfolg schließlich seiner nicht vorhergesehenen Fähigkeit, auch Menschen miteinander in Kontakt zu bringen. In der ersten virtuellen Community The Well, die von Stewart Brand 1985 initiiert wurde (vgl. Hafner 2001), waren alle Internetprotagonisten der ersten Stunde Mitglied: Howard Rheingold, John Pierre Barlow, Sherry Turkle u.a. Spätestens seit Howard Rheingold seiner Hommage an „The Well“ den Titel „The Virtual Community“ (Rheingold 1993) gegeben hat, ist es üblich geworden, unter diesem Begriff Gruppenbildungen im Internet zu bezeichnen, die zum Zwecke der Kommunikation, des Spielens und/oder Kollaboration entstehen und demzufolge auch ethnographisch untersucht werden können.

Virtuelle Communities im Internet gibt es inzwischen unzählige, die auf unterschiedlichem technischen Niveau realisiert werden. Im folgenden möchte ich Newsgroups, MUDs und Spielecommunities als Spezialfälle von virtuellen Communities behandeln und aus der weiteren Betrachtung ausschließen, um auf diese Weise das Gesamtfeld wenigstens etwas einzuschränken.

Newsgroups sind reine Diskussionsgruppen, die auch heute noch überwiegend im Usenet angesiedelt sind. Das Usenet ist der älteste Teil des Internet, lange bevor es die graphische Benutzeroberfläche des World Wide Web (WWW) zu Beginn der 1990er Jahre gab. Es war und ist die erste und einfachste Art der Online Kommunikation: Textnachrichten werden an schwarze Bretter („electronic bulletin boards“) geheftet („gepostet“). Dort können diese Textnachrichten, meist Diskussionsbeiträge, von anderen gelesen und beantwortet werden. Steve Bellovin und andere begannen 1979 diese Art der Online Diskussion an der Duke University. Heute stellen die klassischen NewsgroupsServer ungefähr 22.000 solcher Diskussionsgruppen zur Verfügung. Zwar ist es richtig, dass viele klassische Forschungen sich auf diese Newsgroups bezogen haben und sie auch heute noch Gegenstand klassischer 
Kommunikationsanalysen sind (z.B. Burkhalter 1999), dennoch stellen sie im Kontext des World Wide Web spezifische Gruppen dar, weil sie sich ausschließlich über Kommunikation strukturieren. Die anderen Gruppen, die sich im World Wide Web konstituieren, enthalten (auch visuell) mehr Gestaltungselemente.

Die ersten grundlegenden Forschungen zu Communities sind - neben dem Usenet - in MUDs (Multi User Domains oder auch: Dungeons) gemacht worden. Historisch gesehen sind MUDs ein Abfallprodukt der Künstlichen Intelligenzforschung von Richard Bartle und Roy Trubshaw an der Universität von Essex (UK) in den frühen 1980er Jahren. Es handelt sich in der Regel um Spiele, wobei aber nicht nur Kampfspiele entwickelt worden sind, sondern auch sogenannte TinyMUDs, die soziale Interaktion und kooperatives Weltentwerfen als Gegenzug zu den reinen Kampfspielen betonen (vgl. Cady/McGregor 1995, S. 432ff.). Die meisten MUDs dieser Art sind Rollenspiele, in denen eine angenommene, frei definierbare Identität erprobt werden kann (,running your character"). Man kann in virtuellen Welten umherwandern, mit anderen kommunizieren, gefährliche Gegenden und Situationen erkunden, Aufgaben erledigen, Rätsel lösen, andere Mitglieder treffen, kann sich mit ihnen kooperativ durch Landschaften bewegen und auf diese Weise eigene Potentiale erproben.

Die Spieler entwickeln dabei, wie Götzenbrucker (2001) gezeigt hat, eine Identifizierung mit ihrem MUD, so dass man durchaus von einem Vergemeinschaftungspotential, teilweise sogar von einem sozialen Netzwerk sprechen kann, das über die Onlinewelt hinaus für die Mitglieder eine hohe Bedeutung besitzt. Götzenbrucker beschäftigt sich mit den drei Spiele-Communities: Ultima Online, Silberland und Palazzo. In diesen Communities wird durch kommunikative Aushandlungsprozesse eine Parallelwelt mit einer eigenen Ordnung der Dinge kreiert. Sie hat dabei folgende Aspekte im Blick: Analyse der Auftritts-, Anerkennungs- und Abtrittsrituale; Analyse der Demonstration von Gruppenzugehörigkeit; abweichendes Verhalten und Sanktionen; Beschreibung von Gruppen-Interaktionen sowie sprachliche und kommunikative Besonderheiten. Auf diese Weise kann sie die entsprechenden kulturellen Techniken analysieren und Mechanismen der Sozialintegration über gemeinsam verwendete Symbole, gemeinsame Interessen und kreierte Identitäten beschreiben.

In dieser Perspektive kann also gesagt werden, dass MUDs eine spezifische Form von Communities darstellen, weil sie verschiedene Formen von Kommunikation vereinen, soziale Strukturen der Informationspolitik, der Partizipation, Teilgruppenbildung und Kollaboration ausbilden. MUDs ordne ich der Kategorie der Online-Spielecommunities (wie z.B. die StarCraft Community: www.stammkneipe.de) zu, deren Zweck ausschließlich im Spielen besteht. Diese habe ich im folgenden innerhalb der zu betrachtenden Gruppe der virtuellen Communities nicht ins Zentrum gerückt, eben weil es hier thematisch nur um Spielen geht. Die verbleibenden Communities bilden immer noch ein unüberschaubares Gewirr, das - thematisch gesehen - eine extreme 
Bandbreite umfasst: Partner/Kontakte, Fun, Jugend/Familie, Hobby (u.a. Computer), Gesundheit/Körper, Selbsthilfe, Glauben, Beruf/Ausbildung, TV/ Radio/Printmedien, Städte/Reisen, Politik (um nur gängige Bereiche zu nennen). Kulturalität und Sozialität kann in ihnen in komplexerer Weise analysiert werden. Ich möchte mit dieser relativen Eingrenzung nicht ausschließen und nicht bestreiten, dass Analysen kultureller Praktiken auch in Newsgroups, MUDs oder in Spielecommunities aussichtsreich und mit hohem Erkenntnisgewinn durchgeftihrt werden können. Ich möchte damit lediglich das Phänomen virtuelle Communities so einschränken, dass dennoch eine hinreichende Komplexität und Vielschichtigkeit kultureller Praxen in den Blick genommen werden kann. Diese Entscheidung kann durch einen kurzen Blick auf den Stand der Forschung weiter plausibilisiert werden.

\subsection{Zum Stand der Forschung}

Von Mitte bis Ende der 1990er Jahre stand die Erforschung des Online-Kommunikationsverhaltens im Zentrum (Kommunikations- und Interaktionsstudien). Sempsey (1997) gibt einen guten Überblick über die kommunikativen Teilphänomene, die forschungsmäßig interessierten. Das Interesse an der Exploration des Online-Kommunikationsverhaltens hat bis heute angehalten, hat aber auch, so ist die Forschungslage einzuschätzen, wesentliche Resultate, die als gesichert gelten können, erbracht (Frindte/Köhler 1999; Stegbauer 2001). Seit Ende der 1990er Jahre beschäftigt sich eine zunehmende Zahl von Wissenschaftlerinnen und Wissenschaftlern unter sozialwissenschaftlicher Perspektive mit virtuellen Communities in einem umfassenderen Sinne (Smith/Kollock 1999): Nicht mehr nur dem Strategie- und Kommunikationsverhalten gilt die Aufmerksamkeit, sondern dem „Gesamtleben“ einer virtuellen Community mit ihren verschiedenen strukturellen Aspekten (Vergemeinschaftungsprozesse, Partizipation, Qualität sozialer Beziehungen etc.) (vgl. Garton u.a. 1999; Femback 1999), so dass gesagt werden kann, dass auf diese Weise die kulturellen Praktiken in ihrem Zusammenspiel besser erforscht werden können (Döring 1999; Thiedeke 2000; Marotzki 2001). Innerhalb dieses breiten Forschungsfeldes (vgl. auch Mann/Stewart 2000) ist die Online-Ethnographie - das sei hier erneut gesagt - jener Zugang, der sich in ethnographischer Perspektive mit Gruppenbildungen und neuen Vergemeinschaftungsformen im Internet beschäftigt.

\subsection{Strukturmerkmale virtueller Communities}

Obwohl es inzwischen eine unüberschaubare Anzahl virtueller Gemeinschaften gibt, kann man doch feststellen, dass sich identische Strukturmerkmale herausgebildet haben. Im folgenden rekonstruiere ich acht Merkmalsbereiche, die wir an der Otto-von-Guericke-Universität in Magdeburg in Lehrforschungsprojekten über virtuelle Communities herausdestilliert haben. 
Nachdem wir Online-Ethnographien einzelner Communities erstellt hatten, ging es uns darum, fallübergreifende Strukturen zu ermitteln. Zu diesem Zweck haben wir 40 Communities ${ }^{2}$ ausgewählt und sie darauf hin untersucht, wie in ihnen soziale Ordnung aufgebaut, aufrecht erhalten und verändert wird.

\subsubsection{Leitmetapher für die Infrastruktur}

Eine Community muss im Internet ein bestimmtes Aussehen erhalten, das einer Leitmetapher folgt. In der Regel handelt es sich dabei um die Metapher einer Stadt wie beispielsweise bei www.funama.de. Es können auch andere Metaphern gewählt werden, beispielsweise die einer Bibliothek, eines Zimmers oder eines Planetensystems. Wenn aber eine Leitmetapher gewählt worden ist, dann folgen daraus andere Strukturentscheidungen: In einer Stadt gibt es beispielsweise Häuser, in denen sich Wohnungen befinden, die gemietet werden können. Zur Verwaltung einer Stadt gibt es entsprechende Gebäude, z.B. ein Rathaus. Es muss ein Navigationssystem durch die Stadt geben, z.B. in Form eines U-Bahn-Systems wie bei www.funama.de.

\subsubsection{Regelwerk}

Wie in jeder Gruppe, so gibt es auch in virtuellen Communities Regeln, die teilweise vorgegeben, teilweise von den Mitgliedern ausgehandelt werden. Dazu gehört erstens die Regelung des Zugangs. Während bei vielen Communities im Prinzip jeder Mitglied werden kann - die Angabe eines Nicknames und eines Passwortes reichen in der Regel -, gibt es auch Communities, die schärfere Zugangsregelungen haben. Bei Selbsthilfegruppen beispielsweise wird meistens mehr Information vom Beitrittswilligen erwartet, so dass eine explizite Entscheidung über seine Aufnahme oder Nicht-Aufnahme getroffen werden kann. Bei besonders sensiblen Themen sind auch Telefonrückrufe vorgesehen, um möglichst sicher zu sein, dass die vorgegebene Identität des Beitrittswilligen auch der realen entspricht.

Zweitens finden wir in nahezu allen virtuellen Communities ein Gratifikationssystem. Darunter wird ein System der Belohnung für Aktivitäten innerhalb der Community verstanden. In vielen Fällen handelt es sich um ein Punktesystem oder um virtuelles Geld, das man erhält. Interessant ist bei-

2 Das Sample ist nach zwei Gesichtspunkten zusammengesetzt worden: Zum einen haben wir versucht, die Altersgruppen abzudecken: 3 Communities für Kinder, 9 für Jugendliche, 5 für Erwachsene und 3 für alte Menschen. Zum anderen haben wir nach dem Gesichtspunkt der maximalen Kontrastierung Gegenstandsbereiche unterschieden: 5 Communities für Fun, 2 für Familie/Beziehung, 5 für Selbsthilfe, 5 aus dem beruflichen Kontext sowie 3 Selbstmordcommunities. Die Erhebung und Auswertung erfolgte zusammen mit Studierenden in den Jahren 2001 und 2002. 
spielsweise das Anreizsystem in Fumma. Einige Wohnungen verfügen über einen Akku, der voll geladen ist, wenn man einzieht. Durch Aktivitäten in der Stadt (Besuch von Foren, Chats etc.) wird dieser Akku aufgeladen. Bei Inaktivität sinkt die Ladung des Akkus; ist er leer, wird man aus der Wohnung sanft hinausbefördert. Zweifelsohne liegt den meisten Anreizsystemen der Wunsch zugrunde, die Mitglieder an die Community zu binden und sie zu Aktivitäten zu ermutigen. Pädagogisch mag darüber gestritten werden, ob Aktivitäten in einer Gemeinschaft immer durch ein Anreizsystem begleitet werden sollten. Zu konstatieren ist jedoch, dass es kaum Communities gibt, die ohne solche Anreize auszukommen scheinen.

Drittens ist ein Sanktionssystem offensichtlich unabdingbar. Es schützt Teile der Community oder sie als ganzes vor Missbrauch durch Nutzer, die die Absicht haben zu stören, Unfug zu machen oder das Leben in einer Community lahm zu legen. In der Regel handelt es sich um ehrenamtliche Mitglieder, die diese Aufgabe der Kontrolle und Sanktion wahmehmen, die in Foren und Chats präsent sind, um zu sehen, ob dort beispielsweise Gewalt verherrlichende Inhalte gepostet werden. In dem expliziten Regelwerk der jeweiligen Community wird auf dieses Sanktionssystem hingewiesen; in Funama heißt es beispielsweise: Die Community sei keine rechtsfreier Raum: „Insbesondere gelten folgende Verbote auch für den Aufenthalt in unserer Community: Die Verbreitung oder Zugänglichmachung pornographischer Schriften an Jugendliche unter 18 Jahren (§ 184 Abs.1 StGB); die Verbreitung oder Zugänglichmachung pornographischer Schriften, die Gewalttätigkeiten, den sexuellen Mißbrauch von Kindern oder sexuelle Handlungen von Menschen an oder mit Tieren zum Gegenstand haben (§ 184 Abs.1 StGB); Aufforderungen zu Straftaten ( $§ 111 \mathrm{StGB}$ ); Die Verbreitung von Schriften, die zum RassenHass aufrufen oder grausame und unmenschliche Gewalt gegen Menschen in einer Art schildern, die eine Menschenwürde verletzenden Weise zeigen. (§ 131 StGB); jegliche Form vom extremistischer oder religiöser Gewaltverherrlichung“. Die ehrenamtlichen Mitglieder haben die Möglichkeit, Störern Rechte zu entziehen, Pflichten aufzuerlegen, sie zu ermahnen oder sie auch aus der Community auszusperren.

\subsubsection{Soziographische Struktur}

Unter der soziographischen Struktur wird das System der Über- und Unterordnung sozialer Positionen durch Kompetenzen, Zu- oder Aberkennung von Rechten, Pflichten oder durch Anerkennung verstanden. Die soziographische Struktur wird von den Regeln, vom Gratifikations- und Sanktionssystem einer Community gleichsam gesteuert. Diese stellen den Rahmen dar, innerhalb dessen sich soziale Ordnung bildet und reproduziert (Reid 1999). 


\subsubsection{Kommunikationsstruktur}

Bei der Kommunikationsstruktur handelt es sich zunächst einmal um eine technische Struktur. Es geht um die Möglichkeiten, die in einer Community den Mitgliedern zur Verfügung gestellt werden, um untereinander Kontakt aufzunehmen und miteinander zu kommunizieren.

1) In der Regel handelt es sich bei den Kontaktmöglichkeiten um Chats, die teilweise öffentlich, teilweise halböffentlich und in einigen Fällen auch für geschlossene Gruppen reserviert sind. Weiterhin kann man nach zeitunabhängigen Chats, die also jederzeit zugänglich sind, unterscheiden und nach solchen, die zeitgebunden sind (Terminchats). Selbsthilfegruppen treffen sich beispielsweise meistens zu festgelegten Zeiten in Chats. In technischer Hinsicht finden wir eine große Bandbreite von Chats: von einfachen Textchats bis hin zu Graphikchats ist alles auf fast jedem beliebigen technischen Niveau zu finden.

2) Eine weitere gängige Funktionalität ist die E-Mail, die communityintern wie auch aus der Community heraus nach außen, also an Personen, die nicht dieser Community angehören, verschickt werden kann. Die E-Mail-Funktionalität wird auch für communityinterne Informationen verwendet (E-Mail-Liste). Abonnierbare personalisierte Newsletter stellen auch nichts anderes dar als einen über Filtersysteme individuell konfigurierbaren E-Mail-Verteiler, über den man bestimmte Informationen erhalten kann.

3) Eine weitere Standardfunktionalität ist das Board oder auch Forum. Ein Forum kann im Sinne eines Nachrichtenbrettes verwendet werden, d.h. es werden lediglich Postings platziert, die aber keine Diskussion auslösen; ein Forum kann aber auch im Sinne von Newsgroups funktionieren, d.h. es können Themen gesetzt werden, die eine umfangreiche Diskussion initiieren können. Funama hat beispielsweise insgesamt vier Foren, zwei allgemeine Nachrichtenbretter, ein Brett für Kleinanzeigen und ein Stammtisch-Brett. Empirisch ist zu beobachten, dass in verschiedenen Communities Foren und Chats in unterschiedlicher Weise angenommen werden. In einer Selbstmord-Community wird man beispielsweise eine starke Dominanz der Forenkommunikation beobachten, in reinen Fun-Communities werden Foren, sofern sie vorhanden sind, eher im Sinne von schwarzen Brettern fungieren (zum einmaligen Posten einer Information), ansonsten wird eine starke Dominanz von Chat-Kommunikation zu verzeichnen sein.

4) Weitere Kommunikationsfunktionalitäten, auf die ich an dieser Stelle aber nicht weiter eingehen möchte, sind ICQ, Instant-Messenger und SMS. 


\subsubsection{Informationsstruktur}

In jeder Community werden Informationen zur Verfügung gestellt. Die Frage für die ethnographische Forschung lautet dann: Von wem werden welche Informationen für wen in welcher Form zur Verfügung gestellt. Je nach Gegenstandsbereich der Community werden wir Link-Strukturen und -Sammlungen finden, die auf entsprechende Seiten verzweigen. Funama hat beispielsweise einen „Fresseraum“, in dem wir Links zu hunderten von Tageszeitungen finden. In ambitionierten themenorientierten Communities werden umfangreiche Datenbanken implementiert sein, die mit einem hohen, teilweise professionellem Aufwand gepflegt werden. Die Informationsstruktur kann in Einzelfällen auch mit Lemarenen kombiniert sein, also kleinere E-Leaming-Funktionalitäten enthalten (beispielsweise www.foraus.de).

\subsubsection{Präsentationsstruktur}

1) Die Präsentationsstruktur einer Community umfasst zunächst das Identitätsmanagement. Das kann recht einfach gehandhabt werden, indem beispielsweise nur Nickname und einige wenige selbst gewählte Eigenschaften in der sogenannten IdentityCard eingetragen werden. Es kann aber auch sehr aufwendig betrieben werden, wie beispielsweise in www.cycosmos.de.

\section{Das Beispiel Cycosmos}

Neue Mitglieder treten dort in die Gemeinschaft ein, indem sie sich zunächst selbst eine virtuelles alter ego erschaffen: „Erfinde Dich neu und gib Dich so, wie Du schon immer sein wolltest. Kreiere Identität neu - alles anonym - keiner weiß, wer Du bist. Lerne neue Leute kennen, finde neue Freunde. Definiere Dich neu und wage den Sprung in eine neue Dimension. Du wirst staunen, was für Überraschungen darauf warten, von Dir entdeckt zu werden. Just try it!“ Die Wahl des Nicknames stellt ein zentrales Moment des Überganges in die virtuelle Existenz dar und ermöglicht dem Bewohner der Community Anonymität, um unterschiedliche Facetten des eigenen Selbst und von Sozialität zu explorieren. Mit der zu kreierenden Identität lässt sich entweder die Alltagsidentität verdoppeln oder es kann auch nur ein Teilselbst in die Welt der Simulation übertreten. Wenn das Letztere der Fall ist, werden nur einzelne Facetten der Persönlichkeit im virtuellen Raum präsentiert. Prinzipiell besteht die Möglichkeit, die virtuelle Existenz komplett von der Alltagsexistenz abzukoppeln. Es eröffnen sich Spielräume für die Übernahme vollständig neuer Rollen und neuer sozialer Beziehungen (vgl. Turkle 1995).

Die Schaffung einer virtuellen Identität bedeutet eine Redefinition der eigenen Identität und Leiblichkeit. Die einzelnen Bewohner von Cycosmos (also die sogenannten Cycosrnonauten) entwerfen ein komplexes Muster für 
ihr virtuelles Selbst, das sich aus einer Vielzahl dezentralisierter Komponenten zusammensetzt. Die anzufertigende Selbstbeschreibung erfolgt zunächst graphisch. Es entsteht dabei eine bildliche Darstellung der Identität im Cyberspace, der sogenannte Avatar oder auch das Handle. Der Begriff Avatar bezeichnet die optische Umsetzung eines Pseudonyms in 3D-Chats. Gängige Formen der Avatare im Netz sind: Fotos der Anwender; Bilder einer Person, die der Nutzer gerne sein möchte; Standard-Avatare; oder in Cycosmos: die Herstellung eines Avatars aus dem Baukasten mit einer Reihe von geschlechtsspezifischen Komponenten: Körper, Kopf, Haar, Augen, Gesicht und Accessoires. Im Ergebnis dieses Gestaltungsprozesses entsteht also gewissermaßen ein virtueller Leib. Zum Identitätsdesign gehört neben der Definition der virtuellen Körperlichkeit aber auch die Festlegung von weitergehenden Persönlichkeitseigenschaften. Dem Avatar werden bestimmte Interessen und Vorlieben zugeschrieben. Die entscheidenden Merkmale werden über die Auswahl von Aktivitäten, Charaktereigenschaften, Interessen, Hobbies und Präferenzen bei Musik, Filmen und in der Literatur definiert. Daneben besteht die Möglichkeit, über ein Statement die individuelle Weitsicht sinnspruchartig zu verdichten.

Das angelegte Profil ist nicht statisch, sondern hochgradig wandlungsfähig. Generell besteht die Möglichkeit zur Schöpfung, Neuschöpfung und Veränderung der virtuellen Identität. Körper, Gesichtsausdruck, Haare etc. lassen sich jederzeit per Mausklick redefinieren und so entweder alltagsweltliche Veränderungen in die virtuelle Welt übersetzen bzw. andere Möglichkeiten der Existenz oder bestimmte Facetten des Selbst erproben. Die Konstruktion der virtuellen Identität folgt in Cycosmos vorgegebenen Kategorien und Persönlichkeitsmerkmalen. Es liegen also gewisse Restriktionen vor. Ein Beispiel dafür ist die binäre Codierung durch die vorgegebenen Geschlechter. Es ist lediglich die Wahl zwischen männlich/weiblich möglich. Auch Charaktereigenschaften usw. sind nicht frei zu definieren, sondern müssen aus einem vorgefertigten Set übernommen werden. Insofern lässt sich von einer Digitalisierung der Persönlichkeitseigenschaften sprechen.

Das Resultat aus den Interessen und Charakterzügen, die dem Avatar zugeschrieben werden und den kommunikativen Bezügen, mit denen dieser vernetzt wird, bildet die Identitätskarte (die sogenannte ID-Card). Sie hat bereits auf den ersten Blick Ähnlichkeit mit einer Homepage, ist jedoch nicht im gesamten WWW zugänglich, sondern nur für Mitglieder von Cycosmos. Durch diese Homepage führen die Avatare ein gewisses Eigenleben; sie sind in der virtuellen Welt präsent, existieren dort weiter, auch wenn die User offline sind. Cycosmonauten bleiben über ihre Homepage, die von jedem anderen Bewohner von Cycosmos angesehen werden kann, im virtuellen Raum präsent. Kommunikative Aktivitäten, die an den Avatar adressiert sind, werden in Form von E-Mails und elektronischen Ansichtskarten gespeichert.

2) Neben dem Identitätsmanagement wird zudem oftmals dem Nutzer die Möglichkeit gegeben, eine eigene Homepage zu gestalten, mit der er 
sich im Internet präsentieren kann. Diese Homepage hat oft die Funktion einer erweiterten Identity-Card, denn auf dieser ist ein Link der Homepage verzeichnet, so dass man von dort zur Homepage direkt gelangen kann. Aus dem Inhalt und der Art und Weise, wie diese Homepage gestaltet ist, lassen sich dann weitere Rückschlüsse auf den Nutzer ziehen.

3) Neben öffentlichen Arenen verfügen die meisten Communities auch über halböffentliche und über private Arenen. Ein Beispiel für private Arenen sind Wohnungen, die man sich in Cybercities mietet. Sie können in der Regel individuell eingerichtet werden, „Freunde“ und „Feinde“ können definiert und Freunde können in die Wohnung beispielsweise zum Chatten eingeladen werden. Die privaten Arenen dürfen nicht unterschätzt werden. In Funama (www.funama.de) haben wir beispielsweise eine Untersuchung durchgeführt, die sich mit der Frage beschäftigte, in welchen Bereichen der Community sich die eingeloggten Mitglieder befinden. Auf die technischen Details, wie diese Messungen über den Zeitraum von einer Woche im Mai 2002 durchgeführt wurden, gehe ich an dieser Stelle nicht ein. Das Ergebnis darf aber als repräsentativ gelten. Zum Zeitpunkt der Stichprobe, die an einem Wochentag gegen 18 Uhr gezogen wurde, waren rund 800 Mitglieder in der Community. In der eigenen Wohnung hielten sich 33,5\% auf, mit der eigenen Mailbox, die von der eigenen Wohnung aus zugänglich ist, waren $23,5 \%$ beschäftigt, in öffentlichen Chats waren $21 \%$, in den Bereichen der Community, die man als Spiel und Spaß zusammenfassen kann, hielten sich 12,\% auf und der Rest von 10\% befand sich auf verschiedenen anderen Seiten (z.B. Eingangsseite, Verwaltung, z.B. Rathaus etc.). Das bedeutet, dass zum Zeitpunkt der Stichprobe 57\% der Nutzer sich in privaten Bereichen aufhielten bzw. sich mit nichtöffentlichen Kommunikationsaktivitäten beschäftigten. Daraus darf der Schluss gezogen werden, dass in Funama - und ich würde nach meinen Erfahrungen vermuten, dass das für sehr viele Communities gilt - ein Großteil der sozialen Aktivitäten sich in Mikrozonen (private Wohnung, [definierter] Freundeskreis) abspielt.

\subsubsection{Partizipationsstruktur}

Die Partizipationsstruktur einer Community regelt den Grad der Mitbestimmung, den die Teilnehmer an der Gestaltung der Community haben. Die schwächste Form der Mitbestimmung ist ein Vorschlagswesen. Im schon erwähnten Pressezentrum von funama können beispielsweise Tageszeitungen vorgeschlagen werden, auf deren Seiten ein Link gelegt werden sollte. Etwas stärker sind Communitystrukturen, die es erlauben, Interessengruppen (,interest groups“) durch die Teilnehmer selbst einzurichten. In Cycosmos existierten im Jahre 2000 beispielsweise 120 interest groups. Das Spektrum reicht über Philosophie, Science fiction, Literatur, Wissenschaft bis zu Liebeskummer und Fetisch. Eine neue interest group kann eingerichtet werden, 
wenn sich 30 Akteure zusammentun und dies beantragen. Eine elaborierte Partizipationsstruktur können sicherlich jene Communities aufweisen, die ein demokratisches System der Repräsentation implementiert haben. Ein gutes Beispiel dafür kommt aus dem Bereich der Politiksimulation: www.dol2day.de ist eine der bekanntesten deutschen Politik-Communities. Es ist dort möglich, Parteien zu gründen, Internet-Kanzler zu werden und auf diese Weise die „Politik“ der Community mitzugestalten. Deliberation im öffentlichen Raum und ein konsequentes Entscheidungswesen garantieren hier eine gut ausgebaute Partizipationsstruktur.

\subsubsection{Verhältnis Online-Offline}

Dieser Aspekt bezieht sich erstens darauf, ob in der Community strukturelle Vorkehrungen getroffen worden sind, die es ermöglichen oder sogar fördern, dass Online-Beziehungen offline weitergeführt werden können. Möglichkeiten der Rückbindung an die Alltagswelt der Nutzer sind oben schon genannt worden. Über SMS und Mails, die an die E-Mail-Adressen und Handys außerhalb der Community verschickt werden, können Verabredungen im alltäglichen Leben getroffen werden. Ein weiteres strukturelles Element, das diese Kontaktart erleichtert, ist der Eintrag der Location in die Identity Card, also der Ort, an dem der Nutzer wohnt. Auf diese Weise kann schnell festgestellt werden, wer im gleichen Ort wohnt, so dass eine offline-Kontaktaufnahme ermöglicht wird. Die besten Beispiele dafür finden sich in jenen Communities, die explizit solcher Kontaktaufnahme und Beziehungsanbahnung dienen, beispielsweise www.single.de. Nicola Döring (2000) hat in ihrer Arbeit „Romantische Beziehungen im Netz“ Prozesse der Annäherung, des Kennenlernens und der Beziehungsentwicklung im Internet untersucht und sich dabei u.a. auch für die Phasen interessiert, in denen die reinen Online-Kontakte in real-lebensweltliche Kontakte übergehen.

Das Strukturmerkmal „Verhältnis Online-Offline“ bezieht sich aber nicht nur auf solche gewollten oder ungewollten spill-over-Effekte, sondern beinhaltet zweitens auch häufig eine Servicestruktur, die meistens kommerzielle Anteile aufweist. Links zu Arbeitsämtern, Büchereien oder Angaben von Adressen, von Telefonnummern verweisen den Nutzer einfach weiter zu entsprechenden Institutionen, zu denen er dann anders als online Kontakt aufnehmen kann. Links zu Sparkassen, zu Einkaufsmöglichkeiten haben dagegen hochgradig kommerzielle Anteile. Für die meisten Communities gilt das Merkmal einer Semikommerzialisierung. Fast alle müssen irgendwann die Frage des Geschäftsmodells und damit die der Finanzierung stellen. Exemplarisch kann dies an der Geschichte der ersten Community The Well nachvollzogen werden (vgl. Hafner 2001). Diese Geschäftsmodelle haben ganz unterschiedlichen Charakter. Entweder werden sie von Institutionen, wie z.B. Rundfunkanstalten, finanziert, finanzieren sich über Werbung, nehmen einen monatlichen Mitgliederbeitrag, bieten einen Grundservice frei an, aber wei- 
tergehende Leistungen sind dann kostenpflichtig oder sie bieten Firmen die Möglichkeit, in der Community direkt mit Einkaufsfunktionalitäten präsent zu sein, wie z.B. Versicherungsuntemehmen und Sparkassen.

So weit zu den acht Merkmalsbereichen von Communities. Ihnen kommt zum einen ein hoher heuristischer Wert bei der Analyse weiterer Communities zu. Zum anderen ist diese Kernstruktur, wie die Merkmalsbereiche zusammenfassend auch genannt werden können, dann wichtig, wenn Communities praktisch gestaltet werden sollen. Bei allen Merkmalen gibt es pädagogischen Handlungsund Entscheidungsspielraum. Am Beispiel der Gestaltung eines Gratifikations- und Sanktionssystems hatte ich die grundlegende pädagogische Problematik schon angedeutet. An der Universität Magdeburg bereiten wir zusammen mit Informatikern, Psychologen, Politikwissenschaftlern und Philosophen einen Forschungsverbund vor, innerhalb dessen eine eigene Community (zunächst für Studierende) entwickelt und realisiert werden soll.

\section{Schlussbemerkung}

Vergegenwärtigt man sich neben der enorm hohen Anzahl an Communities im Internet auch die Mitgliederzahlen beispielsweise jener Communities, die für Jugendliche attraktiv sind, dann erstaunen die Zahlen: funama, auf die ich mich in dieser Arbeit mehrfach bezogen habe, hat 120.000 Mitglieder (etwa die gleiche Anzahl besuchen die Community als Gast), Cycosmos hatte im Jahre 2000 ungefähr 450.000 Mitglieder und Metropolis 1,2 Millionen. Ich will an dieser Stelle nicht Vermutungen über mögliche Gründe für diese hohen Zahlen anstellen, sondern lediglich festhalten, dass sich ganz offensichtlich das, was wir soziale Realität nennen, in enormer Weise durch virtuelle Anteile - um es einmal so zu formulieren - erweitert hat. Menschen beginnen, einen Teil ihres Lebens offline und einen anderen Teil online zu organisieren. Die wachsende Bedeutung des Internet für Jugendliche ist beispielsweise ein Thema und Ergebnis der 14. Shellstudie „Jugend 2002“. Sibylle Picot und Michaela Willert beschäftigen sich dort allgemein mit dem enormen Engagement, das Jugendliche im Internet zeigen (vgl. Picot/Willert 2002). Wie gesagt: allgemein und nicht nur bezogen auf Communities, wie ich es hier getan habe. Virtuelle Communities sind neue kulturelle Räume. Es bleibt eine Aufgabe der Forschung, in den nächsten Jahren genauer herauszufinden, wie diese kulturellen Räume strukturiert und welcher Art die kulturellen Praktiken sind. Qualitative Sozialforschung und erziehungswissenschaftliche Biographieforschung werden daran in Form von Online-Ethnographien einen wesentlichen Anteil haben können. 


\section{Literatur}

Bandilla, W.: WWW-Umfragen - Eine alternative Datenerhebungstechnik für die empirische Sozialforschung? In: Batinic, B. u.a. (Hrsg.): Online Research. Methoden, Anwendungen und Ergebnisse. Göttingen u.a. 1999, S. 9-20.

Batinic, B. u.a. (Hrsg.): Online Research. Methoden, Anwendungen und Ergebnisse. Göttingen u.a. 1999.

Burkhalter, B.: Reading race Online: discovering racial identity in Usenet discussions. In: Smith, MVKollock, P. (Eds.). Communities in Cyberspace. London and New York 1999, S. 60-76.

Cady, G. H./McGregor, P.: Mastering the Internet Second Edition. San Francisco u.a. 1995.

Castells, M.: The Rise of the Network Society. The Information Age: Economy, Society and Culture. Volume 1. Oxford, UK1996.

Döring, N.: Sozialpsychologie des Internet. Die Bedeutung des Internet für Kommunikationsprozesse, Identitäten, soziale Beziehungen und Gruppen. Göttingen 1999.

Döring, N.: Romantische Beziehungen im Netz. In: Thimm, C. (Hrsg.): Soziales im Netz. Sprache, Beziehungen und Kommunikationskulturen im Internet Wiesbaden 2000, S. 39-70.

Fernback, J.: There is a There There: Notes toward a Definition of Cybercommunity. In: Jones, St. (Ed.): Doing Internet Research. Critical Issues and Methods for Examining the Net. London u.a. 1999, S. 203-220.

Friebertshäuser, B.: Fremde Lebens weiten verstehen - Ethnographische Feldforschung und Kulturanalysen in der Erziehungswissenschaft. Magdeburg 2000 (Habilitationsschrift Manuskript).

Frindte, W./Köhler, Th.: Kommunikation im Internet. Frankfurt a.M. 1999.

Garton, L./Haythomthwaite, C./Wellmann, B.: Studying On-Line social Networks. In: Jones, St. (Ed.): Doing Internet Research. Critical Issues and Methods for Examining the Net. London u.a. 1999, S. 75-106.

Götzenbrucker, G.: Soziale Netzwerke und Internet-Spielewelten. Eine empirische Analyse der Transformation virtueller in realweltliche Gemeinschaften am Beispiel von MUDs. Wiesbaden 2001.

Hafner, K.: The Well. A Story of Love, Death \& Real Life in the Seminal Online Community. New York 2001.

Hine, Ch.: Virtual Ethnography. In: International Conference: 25-27 March 1998, Bristol, UK IRISS '98: Conference Papers Proceedings (http://www.sosig.ac.uk/iriss/papers/paperl6.htm, Stand: 20.11.2000).

Jones, St. (Ed.): Doing Internet Research. Critical Issues and Methods for Examining the Net. London u.a. 1999.

Kübler, H.-D.: Das unendliche Ende der Wirkungsforschung: das Wirkungspotential der Medien: behauptet, befürchtet, aber noch immer weitgehend unerschlossen. In: Medien praktisch, Jg. 19,1995, Nr. 3. S. 4-12.

Malinowski, B.: Argonauts of the Western Pacific. New York (E.P.Dutton) 1922/1961.

Mann, Ch./Stewart, F.: Internet Communication and Qualitative Research. A Handbook for Researching Online. London u.a. 2000.

Marotzki, W.: Bildung unter den Bedingungen elektronischer Konfigurationen. In: Nieke, W./Masschelein, J./Ruhloff, J. (Hrsg.): Bildung in der Zeit. Zeitlichkeit und Zukunft - pädagogisch kontrovers. Weinheim 2001, S. 27-38.

Miller, D./Slater, D.: The Internet. An Ethnographie Approach. Oxford/New York 2000.

Nieke, W./Masschelein, JVRuhloff, J. (Hrsg.): Bildung in der Zeit. Zeitlichkeit und Zukunft - pädagogisch kontrovers. Weinheim 2001. 
Paccagnella, L.: Getting the Seats of Your Pants Dirty: Strategies for Ethnographie Research on Virtual Communities. In: Journal of Computer Mediated Communication 3(1) June 1997. (http:// www.ascusc.org/jcmc/vol3/issuel/paccagnella.html, Stand: 8.9.2002).

Picot. S./Willert, M.: Politik per Klick - Internet und Engagement Jugendlicher. In: Deutsche Shell (Hrsg.): Jugend 2002. 14. Shell Jugendstudie. Frankfurt a.M. 2002, S. 221- 414.

Reid, E.: Cultural Formations In text-based Virtual Realities. 1994. (http://home.att.net/ aluluei/ cult-form.htm, Stand: 28.09.2002)

Reid, E.: Hierarchy and power: social control in Cyberspace. In: Smith, MVKollock, P. (Hrsg.): Communities in Cyberspace. London and New York 1999, S. 107-133.

Reips, U.-D. u.a. (Hrsg.): Current Internet Science - trends, techniques, results. Aktuelle Online Forschung - Trends, Techniken, Ergebnisse. Zürich 2000. (http://dgof.de/tband99/, Stand: 25.09.2001).

Rheingold, H.: The Virtual Community. New York 1993.

Sandbothe, M./Marotzki, W. (Hrsg.): Subjektivität und Öffentlichkeit. Kulturwissenschaftliche Grundlagenprobleme virtueller Welten. Köln 2000.

Sempsey, J.: Psyber Psychology: A Literature Review pertainig to the Psycho/Social Aspects of Multi-User Dimensions in Cyberspace. In: Journal of MUD Research. Volume 2, Number 1 (1997). (http://joumal.tinymush.org/ jomr/, Stand: August 1997).

Slevin, J.: The Internet and Society. Oxford 2000.

Smith, M./Kollock, P. (Eds.): Communities in Cyberspace. London New York 1999.

Stegbauer, Ch.: Grenzen virtueller Gemeinschaft. Strukturen interaetbasierter Kommunikationsforen. Wiesbaden 2001.

Thiedeke, U. (Hrsg.): Virtuelle Groppen. Charakteristika und Problemdimensionen. Wiesbaden 2000.

Thimm, C. (Hrsg.): Soziales im Netz. Sprache, Beziehungen und Kommunikationskulturen im Internet. Wiesbaden 2000.

Turkle, S.: Life on the Screen. Identity in the Age of the Internet London 1995.

Vogelgesang, W.: Jugend- und Medienkulturen. Ein Beitrag zur Ethnographie medienvermittelter Jugendwelten. In: Kölner Zeitschrift für Soziologie und Sozialpsychologie, Heft 3 (1994), S. 464-491.

Vogelgesang, E.R.W./Wetzstein, T.A./Winter, R.: Auf digitalen Pfaden. Die Kulturen von Hackern, Programmierern, Crackern und Spielern. Wiesbaden 1991.

Zinnecker, J.: Pädagogische Ethnographie. In: Zeitschrift für Erziehungswissenschaft 3/2000, S. 381-400.

Zurawski, N.: Virtuelle Ethnizität. Studien zu Identität, Kultur und Internet. Frankfurt a.M. u.a. 2000. 